\title{
The role of porosity and solid matrix compressibility on the mechanical behavior of poroelastic tissues
}

\author{
H Dehghani $^{1}$, R Penta ${ }^{2}$ and J Merodio \\ Departamento de Mecanica de los Medios Continuos y T. Estructuras, E.T.S. de caminos, canales y puertos, Universidad Politecnica de \\ Madrid, Calle Profesor Aranguren S/N, 28040 Madrid, Spain \\ 2 School of Mathematics and Statistics, Mathematics and Statistics Building, University of Glasgow, University Place, Glasgow G12 8QQ, \\ United Kingdom \\ E-mail: Raimondo.Penta@glasgow.ac.uk
}

Keywords: Poroelasticity, tumor modeling, homogenization, Biot's modulus, biomimetic materials

\begin{abstract}
We investigate the dependence of the mechanical and hydraulic properties of poroelastic materials on the interstitial volume fraction (porosity) of the fluid flowing through their pores and compressibility of their elastic (matrix) phase. The mechanical behavior of the matrix is assumed of linear elastic type and we conduct a three-dimensional microstructural analysis by means of the asymptotic homogenization technique exploiting the length scale separation between the pores (pore-scale or microscale) and the average tissue size (the macroscale). The coefficients of the model are therefore obtained by suitable averages which involve the solutions of periodic cell problems at the pore-scale. The latter are solved numerically by finite elements in a cubic cell by assuming a cross-shaped interconnected cylindrical structure which results in a cubic symmetric stiffness tensor on the macroscale. Therefore, the macroscale response of the material is fully characterized by six parameters, namely the elastic Young's and shear moduli, Poisson's ratio, the hydraulic conductivity, and the poroelastic parameters, i.e. Biot's modulus and Biot's coefficient. We present our findings in terms of a parametric analysis conducted by varying the porosity as well as the Poisson's ratio of the matrix. Our novel three-dimensional results, which are presented in the context of tumor modeling, serve as a robust first step to (a) quantify the macroscale response of poroelastic materials on the basis of their underlying microstructure, (b) relate the compressibility of the tissue, which can be used to distinguish between benign tumor and cancer, to its microstructural properties (such as porosity), and (c) reveal a nontrivial dependency of Biot's modulus on porosity and compressibility of the matrix, which can pave the way to the optimal design of artificial constructs in terms of fluid volume available for transport of mass and solutes.
\end{abstract}

\section{Introduction}

The mechanical behavior of a solid elastic structure interplaying with fluid percolating its pores can be studied via the Theory of Poroelasticity [1-4]. There exists a large variety of scenarios of interest that can be treated by means of a poroelastic modeling approach, including soil mechanics [5], (bio) artificial constructs [6-8], and biological tissues, such as bone [9], organs, healthy and malignant (tumorous) cell aggregates [10].

Materials characterized by a poroelastic mechanical response exhibit an intrinsically multiscale structure. In particular, the average pore radius, and in turn, the distance between them for an approximately uniform pore distribution (the pore scale), is typically much smaller than the average size of the medium (the macroscale) which is effectively behaving as a poroelastic material.

The upscaling process that translates a pore-scale fluid-structure interaction problem into a macroscale problem governed by the equations of poroelasticity can be carried out by means of either average field 
techniques or asymptotic homogenization, see, e.g. [11] and [12] for a comparison between these two alternative approaches in the context of fluid and solid mechanics, respectively.

The former approach is focused on the derivation of the macroscopic model as such, and relies on suitable relationships between the microscale and macroscopic energy of the system at hand. Models deduced this way can be readily extended to a nonlinear constitutive behavior of the individual phases, however, the coefficients are typically not entirely related to the underlying microstructure (see, e.g. the analytic formulas relating drained and undrained coefficients for interconnected pores reported in [5,13]), and are usually to be determined also exploiting experimental measurements. There also exist simplified micromechanical approaches that provide the poroelastic coefficients for specific geometries, for example when spherical, ellipsoidal, or 'penny-shaped' diluted pores are considered, see, e.g. [14]. The asymptotic homogenization technique (see, e.g. [15-20]) exploits the sharp length scale separation between a fine and a coarse scale to represent the fields in terms of power series of the ratio between them. The latter approach entails, in general, a higher degree of algebraic complexity and cannot be trivially generalized to nonlinear balance equations, however, it provides a precise prescription of the coefficients of the model. These encode information concerning the microstructure as they are provided in terms of pore-scale averages which involve both the properties of the individual phases, and auxiliary variables which are to be computed solving differential problems on the pore-scale geometry. The latter is often assumed to be periodic to allow for actual computations of the coefficients on a small and definite portion of the microstructure.

In this work we focus on a material governed by the equations of poroelasticity and embrace the asymptotic homogenization technique to investigate the impact of the interstitial volume fraction (porosity) and compressibility of the matrix, which is assumed to behave as a compressible linear and isotropic elastic solid, on the relevant parameters that determine the overall mechanical response of the material.

In [21] the authors derive the standard Biot's system of partial differential equations (PDEs) via asymptotic homogenization and provide closed formulas for the coefficients of the model, as well as their associated porescale systems of PDEs. In order to compute the coefficients in practice, we also assume pore-scale periodicity, thus embracing the no-growth limit of [22] (which corresponds to the system of PDEs reported in [21] in nonfrequency form) as a starting point. We perform a parametric analysis via finite element numerical simulations to compute the solution of the relevant pore-scale cell problems (which are of Stokes' and linear elastic type) in three-dimensions for various different values of porosity and Poisson's ratio of the elastic matrix. The microscale geometry comprises an interstitial phase made of three interconnected cylinders, which is invariant with respect to permutation of the three orthogonal axes. As a consequence, it is sufficient to investigate the profile of six independent scalar parameters, namely Young's modulus, shear modulus, Poisson's ratio, Biot's modulus, Biot's coefficient, and hydraulic conductivity, which fully characterize the macroscale mechanical and hydraulic behavior of the medium.

Although our analysis is applicable to a number of physical systems, our chief motivation resides in the analysis of deformable malignant cell aggregates (i.e. tumors). A tumor mass can be viewed as a multiscale deformable system, which is composed of several different constituents (such as collagen fibers and proteins forming the extracellular matrix) and cells which collectively form the so-called interstitial matrix [23, 24]. This matrix plays the role of a fluid (and hence drug) transport barrier which reduces the interstitial fluid volume portion accessible for anti-cancer molecules and also exhibits deformations which are supposed to play a role in the spatio-temporal distribution of the interstitial fluid volume and pressure which in turn drive drug transport in malignant tissues, as highlighted in the analysis [24].

Although it is well-known that the porous structure of tumors, and in general biological tissues, affects the observed blood and drug flow maps, experimental data are typically fitted by using mathematical models (for example of poroelastic and viscoelastic type, see e.g. [25] and [24], respectively) which can be reasonably applicable on the macroscale only, and do not explicitly encode the dependence on the underlying microstructure. Furthermore, there only exists a few measurements related to the interstitial volume fraction [26], which exhibits a large variability depending on the specific tissue type and tumor region.

The hydraulic and mechanical properties of solid tumors have been also shown to be affected by its nature (i.e. malignant versus benign), especially in terms of compressibility [27], although a tumor system is often considered as made of intrinsically incompressible phases in order to reduce the number of parameters to be fitted, as done for example in [25] among many others works.

These arguments motivate the development of a computational analysis that can highlight the role of porosity and solid matrix compressibility on the mechanical and hydraulic properties of poroelastic materials framed in the context of solid tumors. Nonetheless, the framework as such is general with respect to the choice of the parameters and our predictions can also be used to reach target mechanical and/or transport properties of artificial poroelastic constructs. The remainder of the work is organized as follows. In section 2 we briefly introduce the asymptotic homogenization technique and state the poroelastic governing equations at the macroscale together with the definitions of the parameters in terms of the relevant auxiliary variables to be 
computed by solving pore scale cell problems. In section 3 we present and discuss the cell problems which are solved to find the auxiliary variables that are needed to compute the macroscale coefficients of the model. In section 4 we present our results, which are obtained by solving the cell problems described in section 3 by finite elements. In section 5 we present our concluding remarks.

\section{Mathematical description of the model}

The formal derivation of the equation of poroelasticity obtained accounting explicitly for the porous microstructure has been reported for the first time in [21]. The authors start from a fluid structure interaction problem between a linear elastic matrix and a Newtonian fluid phase at the pore-scale and close the resulting system of partial differential equations by enforcing continuity of velocities and stresses across the fluid-solid interface. The asymptotic homogenization technique is then exploited to derive the effective governing equations for the medium at the macroscopic scale. In particular, assuming that the pore scale $d$, where distinct microscopic features of the physical system can be identified, and the macroscale $L$, where only global variations of the fields are relevant, are well separated, microscale and macroscale spatial variations can be decoupled as follows

$$
\begin{gathered}
\epsilon=\frac{d}{L} \ll 1, \\
y=\frac{x}{\epsilon} .
\end{gathered}
$$

In (1), $\epsilon$ is a small scale separation parameter, whereas in (2) $\boldsymbol{x}$ and $\boldsymbol{y}$ read as the two formally independent macroscale and microscale spatial variables, respectively. The problem is then upscaled by following the typical steps of the asymptotic homogenization technique. Every relevant field is represented in power series of $\epsilon$, and each component of the fluid velocity $\boldsymbol{v}$, pressure $p$ and elastic displacement $\boldsymbol{u}$, as well as the elastic properties of the matrix, are assumed to be functions of both $\boldsymbol{x}$ and $\boldsymbol{y}$. The system is non-dimensionalized with respect to appropriate characteristic quantities in terms of length scales and velocity fields. In particular, the characteristic (relative) fluid velocity which is used is typically proportional to $d^{2}$ to reflect the fact that the (relative) fluid velocity inside the pores should (approximately) exhibit a parabolic profile, see, e.g. [22, 28, 29] for details concerning non-dimensionalization for porous media flow via asymptotic homogenization. This is indeed formally equivalent to the so-called ' $\epsilon$ viscosity scaling', which is the correct scaling to obtain Darcy's and poroelasticity equations from the microstructure [20-22].

Then, equating the coefficients of the same power of $\epsilon$ for $\epsilon=0,1, \ldots$ in the resulting system of multiscale PDEs leads to a number of differential conditions. These are subsequently exploited to reach a closed differential problem for the leading (zeroth) order components, which represents the homogenized limit for $\epsilon$ approaching zero. The coefficients of the model retain information on the microstructure as they are to be computed by solving pore-scale differential problems which are arising from the upscaling process. The final set of macroscale equations accounts for the effective poroelastic response of the material in terms of average (relative) fluid velocity, pressure, and elastic displacement. Assuming that inertia can be neglected ${ }^{3}$ and accounting for intrinsic incompressibility of the fluid phase (as it is commonly accepted for biological tissues), the governing system of PDEs in the macroscale domain $\Omega \subset \mathbb{R}^{3}$ reads

$$
\left\{\begin{array}{l}
\nabla_{\boldsymbol{x}} \cdot \tau_{E}=0 \\
\tau_{E}:=\tilde{\mathbb{C}} \nabla_{\boldsymbol{x}} \boldsymbol{u}^{(0)}-\tilde{\alpha} p^{(0)} \\
\dot{p}^{(0)}=-M\left[\tilde{\alpha}: \nabla_{\boldsymbol{x}} \dot{\boldsymbol{u}}^{(0)}+\nabla_{\boldsymbol{x}} \cdot \boldsymbol{w}\right] \\
\boldsymbol{w}=-\mathrm{K} \nabla_{\boldsymbol{x}} p^{(0)}
\end{array}\right.
$$

where $p^{(0)}, \boldsymbol{u}^{(0)}, \dot{\boldsymbol{u}}^{(0)}$, and $\boldsymbol{w}$ are the macroscale pressure, solid displacement, solid velocity, and average fluid velocity (relative to the solid displacement). These equations (which are reported by using a notation similar to [22]) are derived in [21] and shown to coincide with the celebrated Biot's equations of poroelasticity once suitable notation identification are made. Relationship (3) represents the stress balance equation for a medium characterized by the constitutive relationship (4). The latter consists of two terms which account for the effective response of the linear elastic phase and the interstitial pore pressure. Equation (5) represents conservation of mass for a poroelastic medium and relates variations of the interstitial fluid pressure to fluid and solid volume changes. The last equation of the macroscale system of PDEs (6) represents Darcy's law for the relative fluid velocity $\boldsymbol{w}$. The mechanical behavior of the material is therefore fully specified by the effective elasticity tensor

\footnotetext{
${ }^{3}$ This assumption is typically embraced for the analysis of the mechanical behavior of biological tissues although, as far as we are concerned, we consider it for the sake of simplifying the notation only, as we are only computing the coefficients, which are exactly the same even when (linearized) inertia is taken into account.
} 


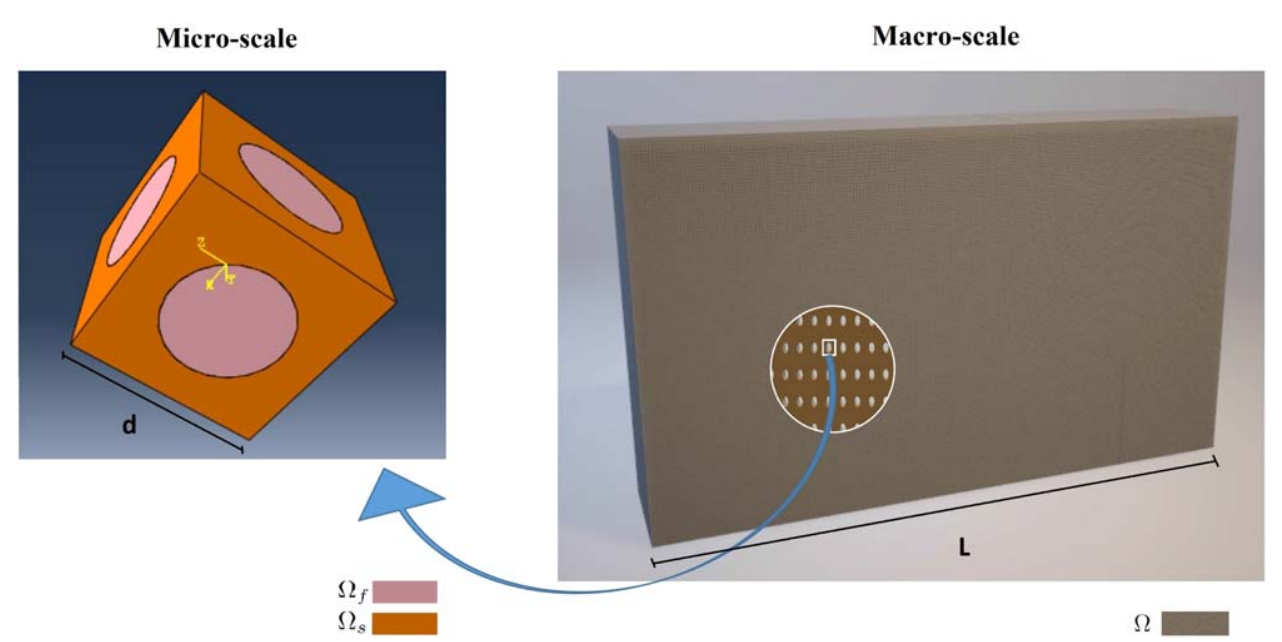

Figure 1. The microstructure, shown on the left, is assumed to be periodic. An example macroscale domain, where pore-scale details are smoothed out, is shown on the right.

$\tilde{\mathbb{C}}$, the hydraulic conductivity K, the Biot's tensor $\tilde{\alpha}$, and the Biot's modulus $M$. According to [21], the above mentioned coefficients can be expressed in terms of suitable averages over the whole microscale domain (spanned by the spatial variable $y$ ), however, suitable regularity assumptions are to be embraced in order to compute the coefficients in practice. Following the traditional approach typical of the asymptotic homogenization literature, and indeed also the suggestions contained in [21], we then assume microscale ( $y$ ) periodicity, as shown in figure 1.

This way, the model coefficients introduced above can be expressed in terms of integral averages over a single periodic cell. The latter are defined via the following cell average operator

$$
\langle\bullet\rangle_{r}=\frac{1}{\left|\Omega_{c}\right|} \int_{\Omega_{r}} \bullet \mathrm{d} \boldsymbol{y} \quad r=f, s,
$$

where $\Omega_{c}$ is the periodic cell domain, with corresponding solid and fluid subdomain $\Omega_{s}$ and $\Omega_{f}$, respectively. The quantities $\left|\Omega_{c}\right|,\left|\Omega_{s}\right|$, and $\left|\Omega_{f}\right|$ are the periodic cell volume and the solid and fluid volume portions, respectively, such that $\left|\Omega_{c}\right|=\left|\Omega_{s}\right|+\left|\Omega_{f}\right|$. In particular, the porosity (that is, the interstitial volume fraction) is given by

$$
\phi=\frac{\left|\Omega_{f}\right|}{\left|\Omega_{c}\right|} .
$$

The relative fluid velocity can then also be expressed as

$$
\boldsymbol{w}=\left\langle\boldsymbol{v}^{(0)}\right\rangle_{f}-\phi \dot{\boldsymbol{u}}^{(0)}
$$

whereas the macroscale coefficients read

$$
\mathrm{K}=\langle W\rangle_{f}, \quad \tilde{\alpha}=\phi \mathbf{l}-\operatorname{Tr}\langle\mathbb{M}\rangle_{s}, \quad \tilde{\mathbb{C}}=\langle\mathbb{C}+\mathbb{C M}\rangle_{s}, \quad M=-\frac{1}{\langle\operatorname{Tr} Q\rangle_{s}} .
$$

The fourth rank tensor $\mathbb{M}$ and the second rank tensors $Q$ and $W$ are to be computed by solving the pore scale periodic cell problems illustrated in the next section.

\section{The cell problems}

We perform our analysis in non-dimensional form, so that the cell problems for the auxiliary variables $\mathbb{M}, Q$, and $W$ are presented and thereafter solved in a unit cubic cell $\Omega_{c}$ (with $\phi\left|\Omega_{f}\right|$ ). The latter comprises the fluid subdomain $\Omega_{f}$, which is given by a cross-shaped cylindrical structure representing a fully interconnected porous media, and the elastic matrix host domain $\Omega_{s}=\Omega_{c} \backslash \Omega_{f}$. This is the simplest possible geometry that accounts for a fully three dimensional flow in a saturated porous media and also ensures that the number of macroscale parameters is kept to a minimum. Figure 2 shows the geometry of the cell as well as the different phases depicted

above, namely the solid, fluid, and their interface $\Gamma=\partial \Omega_{s} \cap \partial \Omega_{f}$. Figure 3 shows a typical computational output related to the solution of a periodic, linear-elastic type cell problem.

We remark that, although the equations of poroelasticity are derived (starting from the microstructure) assuming continuity of velocity and stresses at the start of the asymptotic derivation, the result is indeed a macroscopic system of partial differential equations where the interface between phases is smoothed out (this is 


\section{Geometry of the cell}

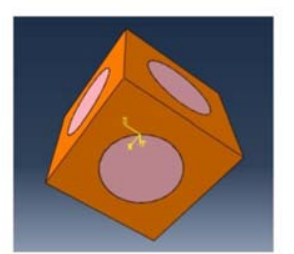

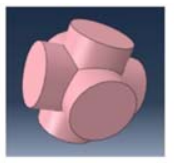

Fluid

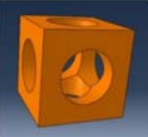

Solid

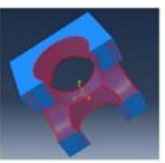

Interface

Figure 2. The cubic cell $\Omega_{c}$ and its corresponding fluid and solid subdomains $\Omega_{f}$ and $\Omega_{s}$, respectively. The interface $\Gamma$ is also highlighted in red.

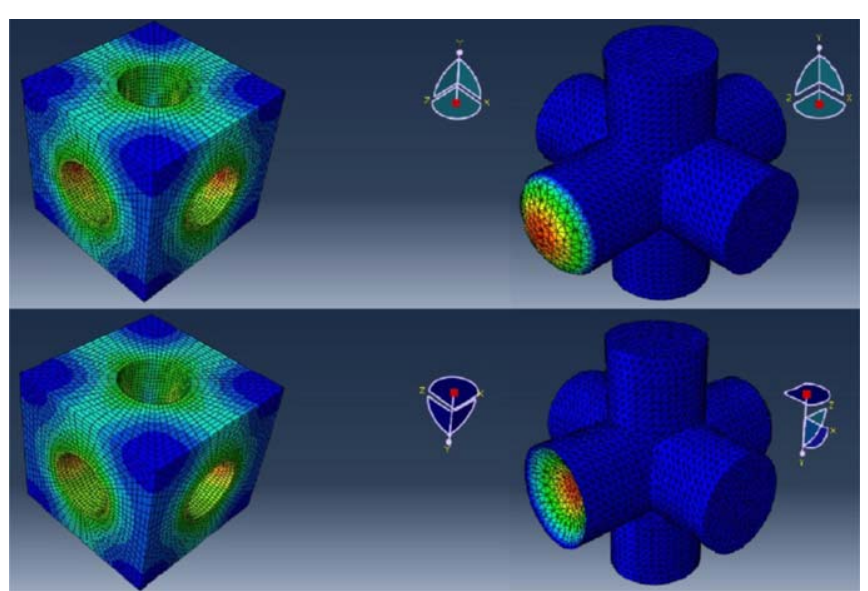

Figure 3. Typical color map showing the periodic solution of the auxiliary displacement computed in Abaqus.

the case when applying asymptotic homogenization to general system of PDEs describing multiphase problems). Once the formulation has been derived, the role of the interface between the solid and the fluid phase is encoded in the relevant cell problems, where the latter actually read, formally, as purely (auxiliary) fluid and solid decoupled problems [18, 21].

Next, we introduce the cell problems for the auxiliary tensor $\mathrm{W}$.

\subsection{The hydraulic conductivity tensor}

The second rank tensor W can be determined by solving the following auxiliary Stokes'-type cell problem on the microscale

$$
\begin{gathered}
\nabla_{y}^{2} \mathrm{~W}^{\top}-\nabla_{y} P+\mathrm{I}=0 \quad \text { in } \Omega_{f} \\
\nabla_{y} \cdot \mathrm{W}^{\top}=0 \quad \text { in } \Omega_{f} \\
\mathrm{~W}=0 \quad \text { on } \Gamma,
\end{gathered}
$$

where $\boldsymbol{P}$ is an auxiliary vector, I is the identity tensor and the (non-dimensional) viscosity of the fluid is set to 1 . The problem is equipped with homogeneous Dirichlet conditions on the interface $\Gamma$ together with periodicity conditions on $\partial \Omega_{f} / \Gamma$ and a suitable uniqueness condition for the vector $\boldsymbol{P}$, e.g.

$$
\langle\boldsymbol{P}\rangle_{f}=\mathbf{0}
$$

without loss of generality.

The system of equations (11)-(13) can be rewritten componentwise as follows

$$
\mu \frac{\partial W_{j i}}{\partial y_{k} \partial y_{k}}-\frac{\partial P_{i}}{\partial y_{j}}+\delta_{i j}=0 \quad \text { in } \Omega_{f}
$$




$$
\begin{array}{cc}
\frac{\partial W_{j i}}{\partial y_{j}}=0 \quad \text { in } \Omega_{f} \\
W_{i j}=0 \quad \text { on } \Gamma,
\end{array}
$$

i.e. the problem (11)-(13) corresponds to three Stokes' problems for $i=1,2,3$. However, since we have chosen a geometry which is invariant with respect to permutation of the three orthogonal axes, we can focus on the problem that corresponds, for example, to $i=1$ only, i.e.

$$
\begin{gathered}
\nabla_{y}^{2} \tilde{\boldsymbol{v}}-\nabla_{y} \tilde{p}+\boldsymbol{e}_{1}=0 \quad \text { in } \Omega_{f} \\
\nabla_{\boldsymbol{y}} \cdot \tilde{\boldsymbol{v}}=0 \quad \text { in } \Omega_{f} \\
\tilde{\boldsymbol{v}}=0 \quad \text { on } \Gamma,
\end{gathered}
$$

where $\tilde{p}=P_{1}$. The above problem formally reads as a periodic Stokes' problem for an incompressible fluid driven by a unit body force directed along $\boldsymbol{e}_{1}$ and the solution $\tilde{\boldsymbol{v}}$ is related to the components of the tensor $\mathrm{W}$ by means of the following identifications

$$
\begin{aligned}
& \tilde{v}_{1}=W_{11}=W_{22}=W_{33} \\
& \tilde{v}_{2}=W_{21}=W_{32}=W_{13} \\
& \tilde{v}_{3}=W_{31}=W_{12}=W_{23},
\end{aligned}
$$

see also [30] where the same argument is used to compute the hydraulic conductivity tensor associated with the capillary compartment of vascularized tumors. This way the full tensor $\mathrm{W}$ and therefore the hydraulic conductivity $\mathrm{K}=\langle\mathrm{W}\rangle_{f}$ can be determined by means of (10). From a numerical standpoint, as we have had no access to Abaqus fluid dynamics modules, we have solved the problem for a linear, isotropic, and incompressible elastic solid (that is equivalent to the incompressible Stokes' problem for the elastic displacement) driven by the same unit body force, whose solution coincides with the problem (18)-(20) once the velocity is identified with the resulting displacement and the viscosity (which is set to 1) with the shear modulus. The dimensional hydraulic conductivity (see, e.g. [22] for the details concerning the non-dimensional analysis of a multiscale poroelastic problem) $\mathrm{K}_{\mathrm{dim}}$ can be obtained by multiplying the result by $d^{2} / \mu$, where $\mu$ is an appropriate (dimensional) fluid viscosity.

\subsection{The effective elasticity tensor and Biot's coefficient}

The fourth rank tensor $\widetilde{\mathbb{C}}$ represents the effective elasticity tensor for a drained poroelastic medium (i.e. it fully characterizes the effective mechanical response when setting the interstitial pore pressure $\left.p^{(0)}=0, \operatorname{cf}(4)\right)$ and, according to relationships (10), is related to both the elasticity tensor of the matrix $\mathbb{C}$ and the auxiliary tensor $\mathbb{M}$ defined as

$$
\mathbb{M}=\xi_{y} \mathcal{A}
$$

where

$$
\xi_{y}(\bullet)=\frac{1}{2}\left(\nabla_{y}(\bullet)+\left(\nabla_{y}(\bullet)\right)^{\top}\right) .
$$

The third rank tensor $\mathcal{A}$ can be determined by solving the following linear elastic-type cell problem

$$
\begin{gathered}
\nabla_{y} \cdot\left(\mathbb{C} \xi_{y} \mathcal{A}\right)=0 \quad \text { in } \Omega_{s} \\
\left(\mathbb{C} \xi_{y} \mathcal{A}\right) \boldsymbol{n}+\mathbb{C} \boldsymbol{n}=0 \quad \text { on } \Gamma \\
\langle\mathcal{A}\rangle_{s}=0,
\end{gathered}
$$

where $\boldsymbol{n}$ is the unit outward vector normal to $\Gamma$. The problem is equipped with periodic conditions on $\partial \Omega_{s} / \Gamma$ and a further condition, for example of the type (28), ensures uniqueness of the solution.

We rewrite the system of equations (26)-(28) componentwise as follows

$$
\begin{gathered}
\frac{\partial}{\partial y_{j}}\left(C_{i j k l} M_{l k \nu \gamma}\right)=0 \quad \text { in } \Omega_{s} \\
C_{i j k l} M_{l k \nu \gamma} n_{j}=-C_{i j \nu \gamma} n_{j} \quad \text { on } \Gamma \\
\left\langle A_{i j k}\right\rangle_{s}=0 \quad \forall i, j, k=1 \ldots 3 .
\end{gathered}
$$

The solution of the problem $M_{l k \nu \gamma}$ can be obtained by solving six elastic-type cell problems by fixing the couple of indices $(\nu, \gamma)$. This way, $M_{l k \nu \gamma}$ in (29) formally represents a strain and for each fixed couple $(\nu, \gamma)$ we have a linear elastic problem equipped with inhomogeneous Neumann interface conditions which can be rewritten componentwise as 


$$
\begin{gathered}
\nu \gamma=11: C_{i j k l} M_{l k 11} n_{j}=-C_{i j 11} n_{j} \quad \text { where } C_{i j 11} n_{j}=f_{i} \\
\nu \gamma=22: C_{i j k l} M_{l k 22} n_{j}=-C_{i j 22} n_{j} \quad \text { where } C_{i j 22} n_{j}=f_{i} \\
\nu \gamma=33: C_{i j k l} M_{l k 33} n_{j}=-C_{i j 33} n_{j} \quad \text { where } C_{i j 33} n_{j}=f_{i} \\
\nu \gamma=23: C_{i j k l} M_{l k 23} n_{j}=-C_{i j 23} n_{j} \quad \text { where } C_{i j 23} n_{j}=C_{i j 32} n_{j}=f_{i} \\
\nu \gamma=13: C_{i j k l} M_{l k 13} n_{j}=-C_{i j 13} n_{j} \quad \text { where } C_{i j 13} n_{j}=C_{i j 31} n_{j}=f_{i} \\
\nu \gamma=12: C_{i j k l} M_{l k 12} n_{j}=-C_{i j 12} n_{j} \quad \text { where } C_{i j 12} n_{j}=C_{i j 21} n_{j}=f_{i}
\end{gathered}
$$

Assuming that the elastic matrix is isotropic at the pore scale we have

$$
C_{i j k l}=\lambda \delta_{i j} \delta_{k l}+\mu\left(\delta_{i k} \delta_{j l}+\delta_{i l} \delta_{j k}\right)
$$

and substituting the relationship (38) into the interface loads (32)-(37) we obtain

$$
\begin{gathered}
\nu \gamma=11: \boldsymbol{f}=\lambda \boldsymbol{n}+2 \mu n_{1} \boldsymbol{e}_{\mathbf{1}} \\
\nu \gamma=22: \boldsymbol{f}=\lambda \boldsymbol{n}+2 \mu n_{2} \boldsymbol{e}_{2} \\
\nu \gamma=33: \boldsymbol{f}=\lambda \boldsymbol{n}+2 \mu n_{3} \boldsymbol{e}_{3} \\
\nu \gamma=23: \boldsymbol{f}=\mu\left(n_{3} \boldsymbol{e}_{2}+n_{2} \boldsymbol{e}_{3}\right) \\
\nu \gamma=13: \boldsymbol{f}=\mu\left(n_{3} \boldsymbol{e}_{1}+n_{1} \boldsymbol{e}_{3}\right) \\
\nu \gamma=12: \boldsymbol{f}=\mu\left(n_{2} \boldsymbol{e}_{1}+n_{1} \boldsymbol{e}_{2}\right),
\end{gathered}
$$

where $n_{1}, n_{2}, n_{3}$ are the components of the unit vector normal to the interface $\Gamma$ and $\boldsymbol{e}_{1}, \boldsymbol{e}_{2}$ and $\boldsymbol{e}_{3}$ are the standard unit vectors in the Cartesian coordinate system. As our input parameters are actually the matrix's Young's modulus $E$ and Poisson's ratio $\nu$, we remind the standard relationships between the latter and the Lamé constants below

$$
\lambda=\frac{E \nu}{(1+\nu)(1-2 \nu)} ; \quad \mu=\frac{E}{2(1+\nu)} .
$$

Considering left and right minor symmetries, Voigt notation can be used to reduce the formally 81 components of the fourth rank auxiliary tensor $\mathbb{M}$ and the effective elasticity tensor $\tilde{\mathbb{C}}$ to 36 and map them in the form of $C_{\alpha \beta}$ and $M_{\beta} \kappa$, where $\alpha, \kappa$, and $\beta=1,2,3, \ldots, 6$. As the solid matrix is assumed to be isotropic, the invariance properties of the geometry dictate that both $\mathbb{M}$ and $\tilde{\mathbb{C}}(\mathrm{cf}(10))$ are cubic symmetric. An example of the resulting cubic symmetric tensors (for porosity $\phi=0.286$, Poisson's ratio $\nu=0.35$ and non-dimensional $E=13.5$ ) is reported below.

$$
\begin{gathered}
\mathbb{M}=\left(\begin{array}{cccccc}
-0.35 & -0.094 & -0.094 & 0 & 0 & 0 \\
-0.094 & -0.35 & -0.094 & 0 & 0 & 0 \\
-0.094 & -0.094 & -0.35 & 0 & 0 & 0 \\
0 & 0 & 0 & -0.167 & 0 & 0 \\
0 & 0 & 0 & 0 & -0.167 & 0 \\
0 & 0 & 0 & 0 & 0 & -0.167
\end{array}\right) \\
\tilde{\mathbb{C}}=\left(\begin{array}{cccccc}
8.59 & 3.23 & 3.23 & 0 & 0 & 0 \\
3.23 & 8.59 & 3.23 & 0 & 0 & 0 \\
3.23 & 3.23 & 8.59 & 0 & 0 & 0 \\
0 & 0 & 0 & 2.97 & 0 & 0 \\
0 & 0 & 0 & 0 & 2.97 & 0 \\
0 & 0 & 0 & 0 & 0 & 2.97
\end{array}\right)
\end{gathered}
$$

In fact, we can conduct the analysis of the elastic moduli in terms of the effective Young's modulus, Poisson's ratio and shear modulus $E_{p}, \nu_{p}$, and $\mu_{p}$, respectively, which are related to the independent components $\tilde{C}_{11}, \tilde{C}_{12}, \tilde{C}_{44}$ via the following relationships, see, e.g. [31]

$$
\begin{gathered}
E_{p}=\frac{\tilde{C}_{11}\left(\tilde{C}_{11}+\tilde{C}_{12}\right)-2 \tilde{C}_{12}^{2}}{\tilde{C}_{11}+\tilde{C}_{12}} \\
\nu_{p}=\frac{\tilde{C}_{12}}{\tilde{C}_{11}+\tilde{C}_{12}} \\
\mu_{p}=\tilde{C}_{44} .
\end{gathered}
$$

Finally, the Biot's tensor given by relationship (10) is actually diagonal as a consequence of cubic symmetry of $\mathbb{M}$. We have

$$
\operatorname{Tr} \mathbb{M}=M_{i j k l} \delta_{l k}=\left(M_{1111}+2 M_{1122}\right) \delta_{i j}
$$


so that the Biot's tensor reads

$$
\tilde{\alpha}=\alpha \mathrm{l},
$$

with scalar Biot's coefficients given by (cf(10)):

$$
\alpha=\phi-\left\langle M_{11}+2 M_{12}\right\rangle_{s},
$$

where the Voigt notation has been used.

\subsection{The Biot's modulus}

The Biot's modulus is defined, according to (10), as

$$
M=-\frac{1}{\langle\operatorname{Tr} Q\rangle_{s}},
$$

where the second rank tensor is given by $Q=\nabla_{y} \boldsymbol{a}$ and $\boldsymbol{a}$ is the solution of the following periodic cell problem

$$
\begin{aligned}
& \nabla_{\boldsymbol{y}} \cdot\left(\mathbb{C} \nabla_{\boldsymbol{y}} \boldsymbol{a}\right)=0 \quad \text { in } \Omega_{s} \\
& \left(\mathbb{C} \nabla_{y} \boldsymbol{a}\right) \boldsymbol{n}+\boldsymbol{n}=0 \quad \text { on } \Gamma \text {. }
\end{aligned}
$$

As previously, the cell problem (55)-(56) formally reads as a linear elastic problem equipped with inhomogeneous Neumman interface conditions on $\Gamma$ and periodic conditions on $\partial \Omega_{s} \backslash \Gamma$, and an additional condition on $\boldsymbol{a}$ is required to ensure uniqueness of the solution, e.g.

$$
\langle\boldsymbol{a}\rangle_{s}=0 .
$$

In the next section, we present the results that are based on the numerical solutions of the cell problems that we have illustrated here. Numerical simulations are performed using the commercial software Abaqus, see also $[32,33]$ for other examples of poroelastic finite element modeling using this software.

\section{Results}

We present our results in terms of effective hydraulic conductivity, poroelastic parameters (Biot's modulus and Biot's coefficient), elastic moduli (Young's and shear modulus) and Poisson's ratio. The cell problems presented in the previous section are solved via finite elements, and the accuracy of the results has been verified performing the sensitivity analysis briefly summarized in the appendix. As the analysis is conducted in non-dimensional form, all the cell problems are solved in the relevant fluid and solid subsets of the cubic unit cell $\Omega_{c}$, i.e. $\Omega_{f}$ and $\Omega_{s}$, respectively. Every parameter is assigned in non-dimensional form and, by considering a suitable non-dimensional analysis (see [22]), the dimensional counterpart of any parameter having the physical dimension of a stress ([Pa]), such as Young's modulus, shear modulus, and Biot's modulus, can be obtained by multiplying the result by a reference value (for example a representative pressure as done in [22]).

Biot's coefficient and Poisson's ratio are non-dimensional, whereas the hydraulic conductivity ([ $\mathrm{m}^{2}$ $(\mathrm{Pa} \cdot \mathrm{s})]$ ) can be obtained by multiplying the non-dimensional result by $d^{2} / \mu$, where $d$ is the characteristic microscale length and $\mu$ the dimensional viscosity of the interstitial fluid. The elastic moduli are computed for the drained case (i.e. by using directly the

effective stress tensor $\mathbb{C}^{\sim}$ ) and also for the undrained case (i.e. when considering the elastic response for a

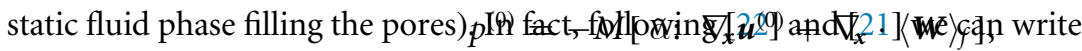

where $\boldsymbol{u}^{(0)}$ and $\langle\boldsymbol{W}\rangle_{f}$ are the solid and fluid displacements.

By using (58) we can write (4) in the form of

$$
\tau_{E}:=(\tilde{\mathbb{C}}+M \tilde{\alpha} \tilde{\alpha}) \nabla_{x} \boldsymbol{u}^{(0)}+(M \tilde{\alpha}) \nabla_{x} \cdot\langle\boldsymbol{W}\rangle_{f} .
$$

The fourth rank tensor

$$
\tilde{\mathbb{C}}+M \tilde{\alpha} \tilde{\alpha}
$$

represents the undrained elasticity tensor of the porous medium, and since in our case $\tilde{\alpha}$ is diagonal, the additional contribution which involves the Biot's modulus and coefficient only affects the effective Young's modulus and Poisson's ratio, as in the case of a macroscopically isotropic poroelastic medium described in [21]. The input parameters are the (non-dimensional) Young's modulus and Poisson's ratio of the matrix $E$ and $\nu$, respectively, and the porosity $\phi$. As highlighted in the Introduction, here we focus on the role of porosity and compressibility of the matrix on the effective hydraulic and mechanical properties of poroelastic materials and therefore the Young's modulus of the matrix is fixed to a reference value, which is set to 13.5. The latter nondimensional value corresponds to a dimensional value of $202.5 \mathrm{mmHg}$, i.e. $\approx 2 \cdot 10^{-3} \mathrm{~Pa}$ (as deduced from the 


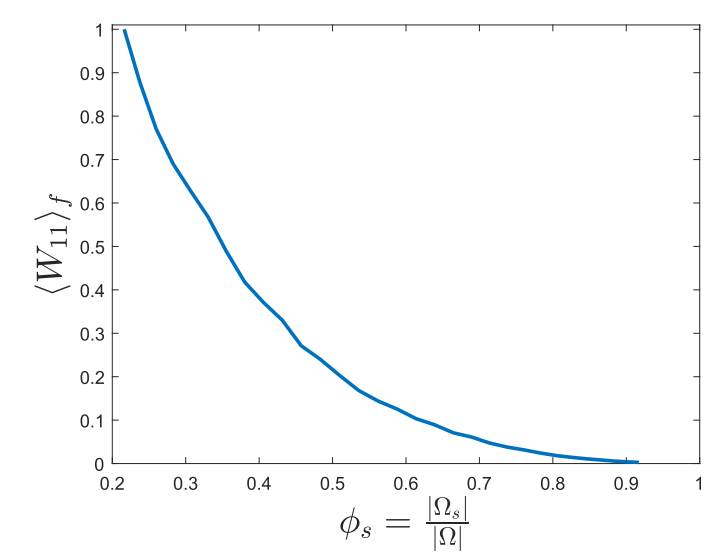

Figure 4. Non-dimensionalized hydraulic conductivity (normalized with respect to its maximum value) vs the solid volume fraction.

Lamé constants reported in [10] for poroelastic tumors), when a dimensional reference (interstitial) pressure of $15 \mathrm{mmHg}$ (see, e.g. [34]) is taken into account.

The parametric analysis is carried out in terms of the porosity and Poisson's ratio of the matrix. In particular, the radius of the three interconnected cylinders is varied from 0.1 to 0.4 with steps of 0.01 , which corresponds to a porosity range $\phi \in[0.083,0.784]$. The profile of every elastic and poroelastic parameter is also evaluated with respect to four different Poisson's ratios, namely, $0.35,0.4,0.45$, and 0.49 , to parametrically explore both the role of tumor compressibility recently reported in [35], and also account for the incompressible case (which is approximated by setting $\nu=0.49$ ). We commence presenting the resulting hydraulic conductivity profile against porosity.

\subsection{Hydraulic conductivity}

The hydraulic conductivity tensor $\mathrm{K}$ is computed as described in section 3 for each value of the cylinders' radius belonging to $(0.1,0.4)$ with steps of 0.01 , so that we obtain the results for the porosity $\phi \in[0.083,0.784]$.

As the driving force of the problem is directed along one fixed direction and the geometry is invariant with respect to permutation of the three orthogonal axes, we expect a diagonal conductivity tensor, i.e.

$$
\mathrm{K}=\left\langle W_{11}\right\rangle \mathrm{I},
$$

which is indeed consistent with our numerical results and also those shown in [30].

Figure 4 shows the hydraulic conductivity $\left\langle W_{11}\right\rangle_{f}$ against the volume fraction of the elastic matrix $\phi_{s}=1-\phi$. We observe a nonlinear drop of $\left\langle W_{11}\right\rangle_{f}$ at decreasing porosity, which is consistent with the parabolic-like profile obtained as a solution to the problem (18)-(20). The values shown are normalized with respect to the maximum value $1.41 \cdot 10^{-2}$, which is attained for a cylinder radius equal to 0.4 (i.e. $\phi \approx 0.784$ ). The minimum value (for a cylinder radius equal to 0.1 , i.e. $\phi \approx 0.08$ ) is $3.8 \cdot 10^{-5}$. The dimensional hydraulic conductivity $K_{\text {dim }}$ is obtained by multiplying the corresponding dimensionless values by $d^{2} / \mu$, where $d$ is the characteristic microscale length and $\mu$ the interstitial fluid viscosity. A physiologically reasonable range is obtained by considering the plasma viscosity $\mu=4 \cdot 10^{-3} \mathrm{~Pa} \cdot \mathrm{s}$ and a typical $d \approx \mu \mathrm{m}=10^{-6} \mathrm{~m}$ (see, e.g. [36]), that yields

$$
10^{-14} \mathrm{~m}^{2} /(\mathrm{Pa} \cdot \mathrm{s}) \lesssim K_{\mathrm{dim}}=\left(d^{2} / \mu\right)\left\langle W_{11}\right\rangle_{f} \lesssim 10^{-12} \mathrm{~m}^{2} /(\mathrm{Pa} \cdot \mathrm{s}) .
$$

The latter corresponds to $10^{-8}-10^{-6}(\mathrm{~cm})^{2} /(\mathrm{mmHg} \cdot \mathrm{s})$ and tumor hydraulic conductivity values reported in the experimental literature typically fall within this range, see for example [25] and [24] among many others. We now proceed by illustrating the profile of the poroelastic parameters against porosity and Poisson's ratio of the matrix.

\subsection{Poroelastic parameters}

The second and fourth rank tensors $Q$ and $\mathbb{M}$ are computed for each considered value of porosity and Poisson's ratio and the scalar Biot's coefficient $\alpha$ and (non-dimensional) Biot's modulus $M$ are obtained via relationships (53) and (54).

Figure 5 shows Biot's coefficient versus porosity for the four different Poisson's ratios. As the porosity approaches 1 , the Biot's coefficient exhibits a monotonically increasing profile which tends to the horizontal asymptote $\alpha=1$ for all Poisson's ratios. The Biot's coefficient physically represents the ratio of macroscale solid to fluid volume changes (at constant pressure), and $\alpha=1$ indeed represents the classical poroelastic upper 


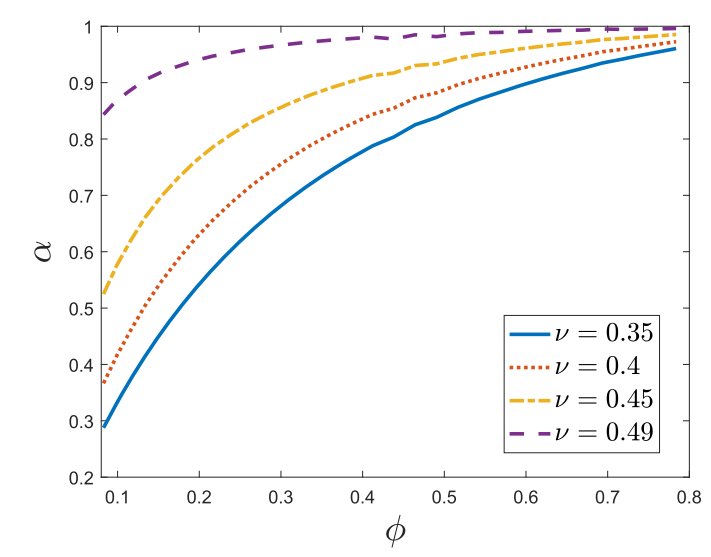

Figure 5. Biot's coefficient versus porosity for four different value of the matrix' Poisson's ratio.

bound for the Biot's coefficient (see, e.g. [37]). The latter is identically reached when both phases are intrinsically incompressible (i.e. $\alpha=1$ for any porosity value when $\nu=0.5$ ). As such, Biot's coefficient approaches 1 at increasing porosity (that is, increasing the incompressible fluid volume fraction and consequently decreasing the compressible elastic volume fraction) and at increasing Poisson's ratio.

Biot's modulus represents the macroscale inverse of the fluid volume variation in response to a variation of the macroscale pore pressure, that is, for a fixed pressure variation, the lower the $M$, the higher the variation of the fluid volume is. Biot's modulus is computed by solving the microscale problem (55)-(56) and then exploiting relationship (54), which is reported below for the readers' convenience

$$
M=-\frac{1}{\langle\operatorname{Tr} Q\rangle_{s}} .
$$

The problem (55)-(56) formally reads as an elastic problem for the auxiliary displacement $\boldsymbol{a}$, with solution driven by the interface load which is directly proportional to the interface normal $\boldsymbol{n}$. The scalar quantity $\operatorname{Tr} Q=\nabla \cdot \boldsymbol{a}$ actually represents the solid volume variation of an elastic solid (characterized by the elastic response of the matrix given by the stiffness tensor $\mathbb{C}$ ) subject to such a load on the interface $\Gamma$ and periodic boundary condition on $\partial \Omega_{s} / \Gamma$. As expected, $\operatorname{Tr} Q=\nabla \cdot \boldsymbol{a}$ exhibits a monotonically decreasing profile towards zero as the Poisson's ratio of the matrix approaches $\nu=0.5$, which is the incompressible limit. In this case, as the Biot's modulus given by (54) is indeed obtained by considering intrinsic incompressibility of the fluid phase, $M$ approaches infinity for every value the porosity, which is consistent with classical poroelasticity. The Biot's modulus profile versus porosity exhibits an interesting and not completely intuitive behavior. In fact, according to our numerical results, $\operatorname{Tr} Q=\nabla \cdot \boldsymbol{a}$ is actually increasing at increasing porosity (i.e. at decreasing solid volume portion where the problem is actually solved), as the problem is solved on the elastic domain $\Omega_{s}$ only and therefore, at fixed Poisson's ratio, volume variations increase. On the other hand, the latter behavior only refers to the drained problem (55)-(56), while the actual effective coefficient accounts for the macroscale response of the material (in terms of fluid variations) and is actually averaged over the relevant solid cell portion $\Omega_{s}$. As a consequence, the average $\langle\operatorname{Tr} \mathrm{Q}\rangle_{s}$ actually approaches zero (and hence $M \rightarrow+\infty$ ) whenever the porosity becomes closer and closer to 1 (that is, when the solid volume fraction approaches zero), while, in general, the Biot's modulus profile versus the porosity is characterized by a definite minimum, and the porosity value at which such a minimum is attained decreases at increasing Poisson's ratio of the matrix $\nu$. The Biot's modulus versus porosity for the four considered values of the Poisson's ratio is shown in figures 6(a)-(d).

Furthermore, since the observed behavior suggests that there is a non-trivial interplay between compressibility of the matrix and porosity, we have also performed a parametric analysis by studying the Biot's modulus profile versus the Poisson's ratio of the matrix. We have considered the same range $\nu \in(0.35,0.49)$ with more refined steps of 0.01 . This analysis has been performed for seven different values of the porosity $\phi$, namely $0.0828,0.1737,0.2864,0.4122,0.5427,0.6694$, and 0.7838 , which correspond to a cylinder radius ranging from 0.1 to 0.4 with steps of 0.05 . The results are shown in figure 7 .

As expected, Biot's modulus is monotonically increasing at increasing Poisson's ratio for every porosity value. As a consequence of the non-trivial dependency on the porosity, the curves representing Biot's modulus versus the Poisson's ratio of the matrix can actually cross each other, i.e same values of $M$ at fixed Poisson's ratio can be obtained for different porosities. The latter behavior is no longer present for very high values of the porosity, as in this case the Biot's modulus is almost monotonically increasing at increasing porosity, see for instance figure 6(d). The results concerning the effective elastic moduli are shown below. 


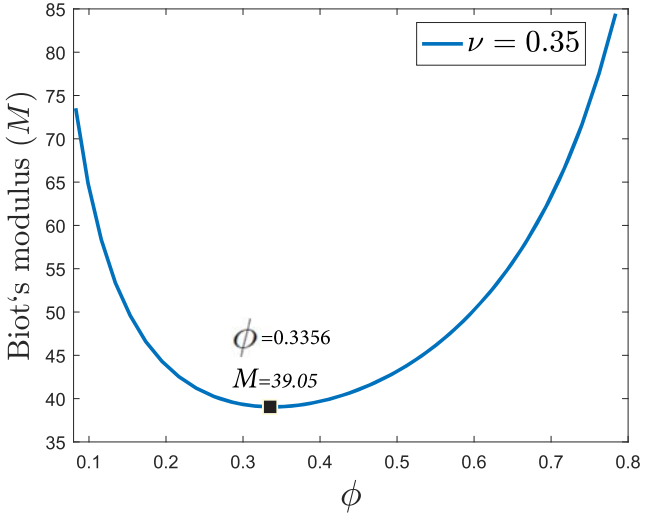

(a) $\nu=0.35$

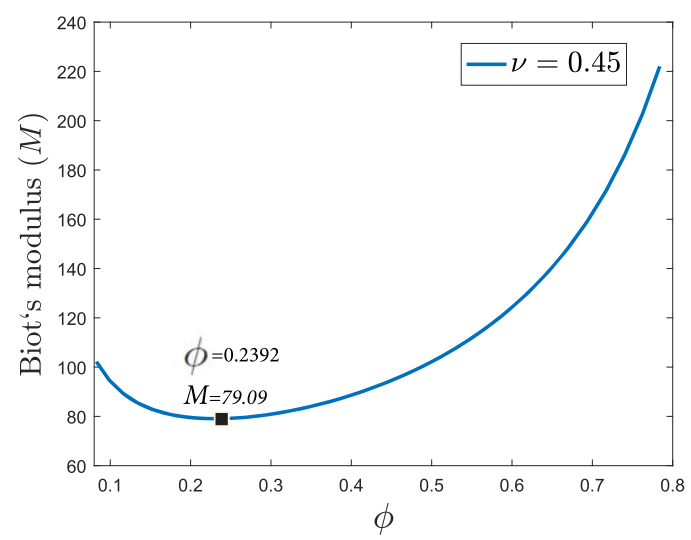

(c) $\nu=0.45$

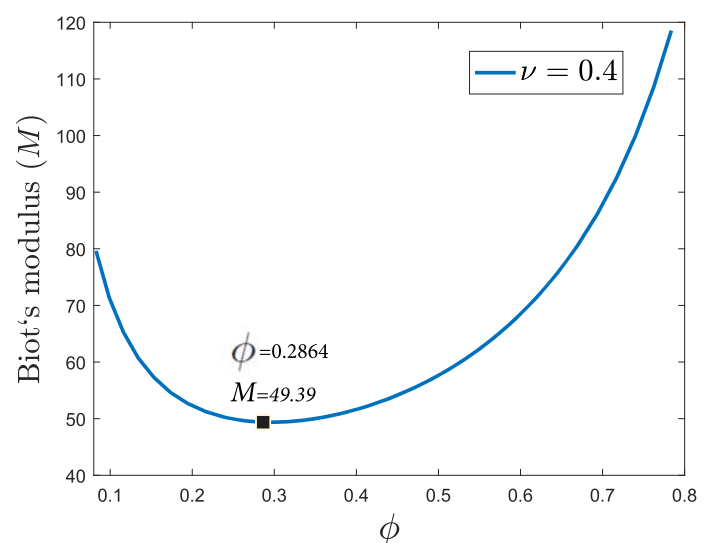

(b) $\nu=0.40$

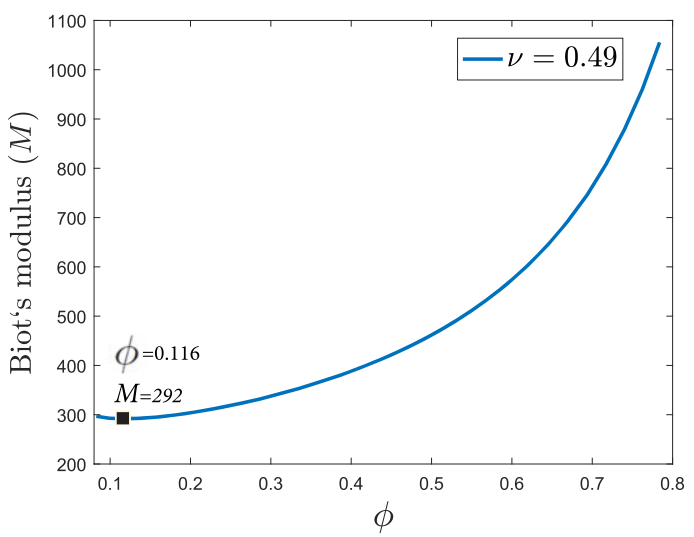

(d) $\nu=0.49$

Figure 6. Non-dimensional Biot's modulus against porosity.

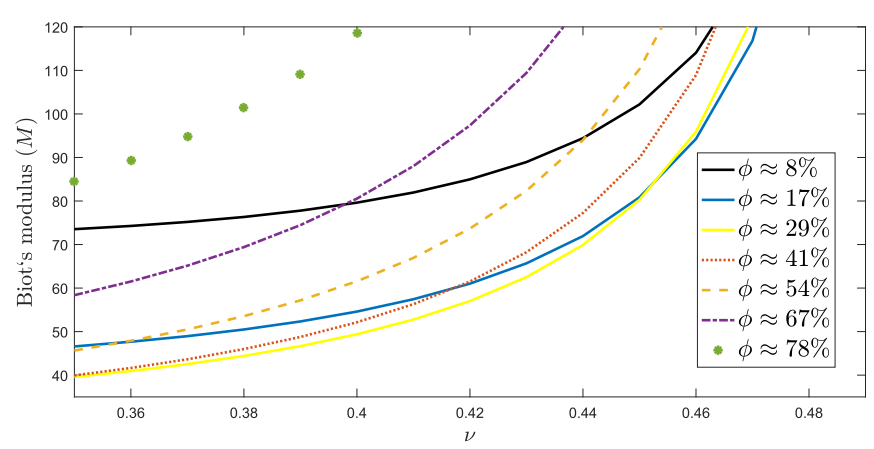

Figure 7. Biot's modulus versus Poisson's ratio of elastic matrix.

\subsection{Elastic moduli}

The drained effective elasticity tensor $\tilde{\mathbb{C}}$ is obtained via relationship (10), which involves both the auxiliary fourth rank tensor $\mathbb{M}$ and the elasticity tensor of the matrix $\mathbb{C}$. The undrained elasticity tensor is computed by adding to $\tilde{\mathbb{C}}$ the contribution related to the poroelastic parameters $M$ and $\alpha(\operatorname{cf}(60))$. Drained and undrained Young's moduli and shear modulus $E_{p}, E_{p s}$, and $\mu_{p}$, respectively, are then obtained from the components of the relevant elasticity tensor by exploiting relationships (48) and (50). The drained and undrained Young's modulus profile versus porosity is shown in figures 8 (a) and (b), while the shear modulus against porosity is shown in figure 9.

Both Young's and shear moduli exhibit a decreasing profile at increasing porosity. The poroelastic material, as expected, becomes more and more compliant towards higher values of the porosity and the drained Young's modulus is slightly lower than the undrained one. This behavior is expected, as the former parameter measures 


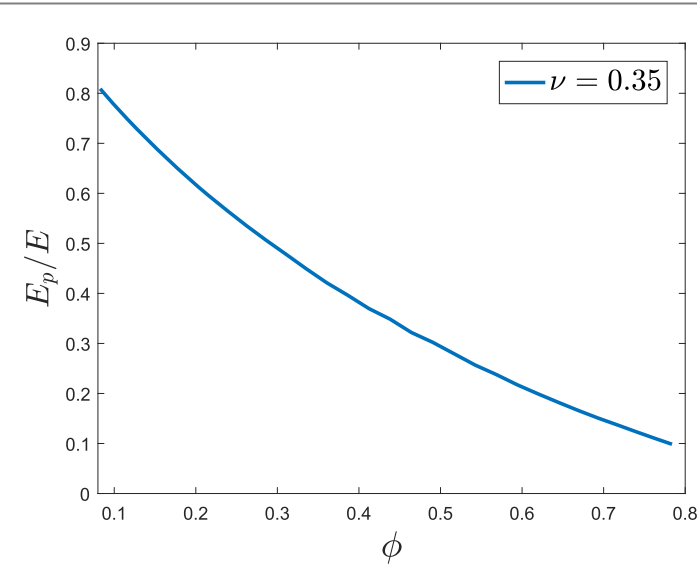

(a) Drained

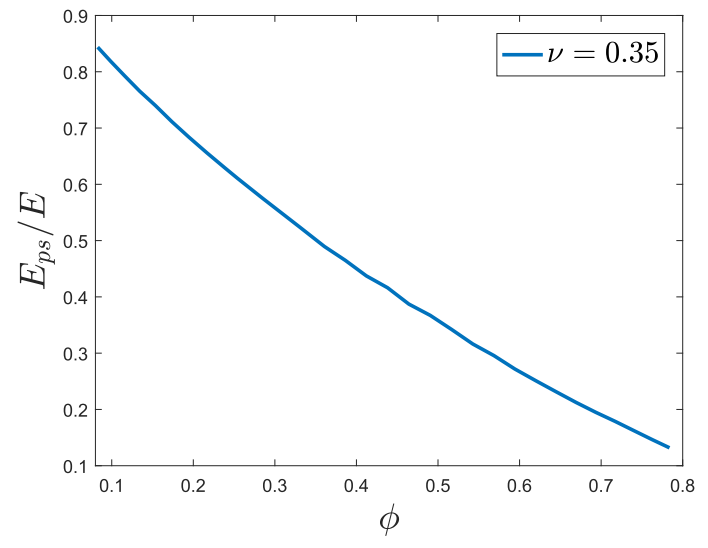

(b) Undrained

Figure 8. Relative non-dimensionalized effective Young's modulus versus porosity. (a) Drained (b) Undrained.

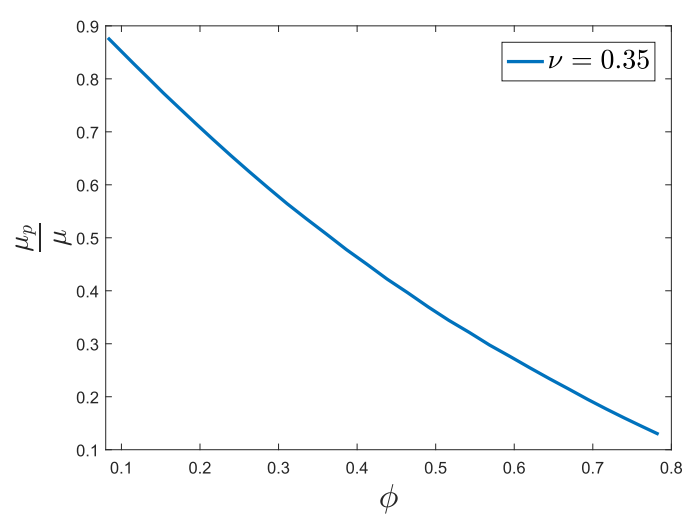

Figure 9. Relative non-dimensionalized effective shear modulus versus porosity.

the macroscale elastic response to axial deformations as though the pores were empty, whereas the latter accounts for the fluid filling the pores (as though the fluid velocity were zero). Thus, the porosity plays a major role in determining the stiffness of poroelastic materials, while the results indicate that the dependency of the elastic moduli on the Poisson's ratio of the solid matrix is non-significant in the investigated range. Therefore, we have only shown the results for $\nu=0.35$, which is our base value. The effective Poisson's ratio, however, strongly depends on the both the Poisson's ratio of the matrix and the porosity, as shown below.

\subsection{Poisson's ratio}

The effective drained and undrained Poisson's ratios $\nu_{p}$ and $\nu_{p s}$ are shown in figures 10 and 11 , respectively. The macroscale drained Poisson's ratio $\nu_{p}$, as expected, decreases at increasing porosity for every investigated value of the matrix' Poisson's ratio, thus reflecting the increased compressibility of the material as a whole at empty pores. Conversely, the macroscale undrained Poisson's ratio $\nu_{p s}$ approaches 0.5 , which corresponds to the incompressible mechanical response, at increasing porosity. This is once again the intuitively expected behavior, as $\nu_{p s}$ measures the macroscale compressibility of the material as though the pores were filled by an incompressible static fluid. In the following section we present our concluding remarks.

\section{Concluding remarks and further perspectives}

In this work, we have performed a three-dimensional analysis of the role of porosity and solid matrix compressibility on the mechanical and hydraulic properties of poroelastic materials. We have carried out numerical simulations by finite elements, making use of parameters that are typical of biological, cancerous tissues. We have started from the equations of linearized poroelasticity and computed the effective coefficients of 


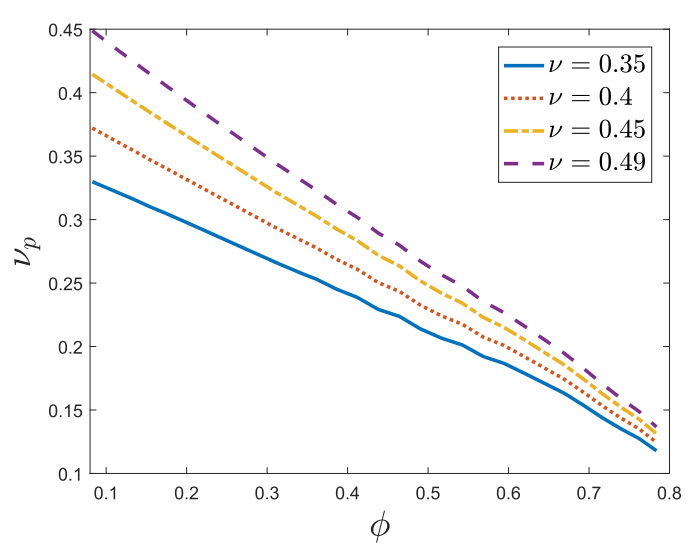

Figure 10. Drained effective Poisson's ratio versus porosity for different Poisson's ratios of the matrix.

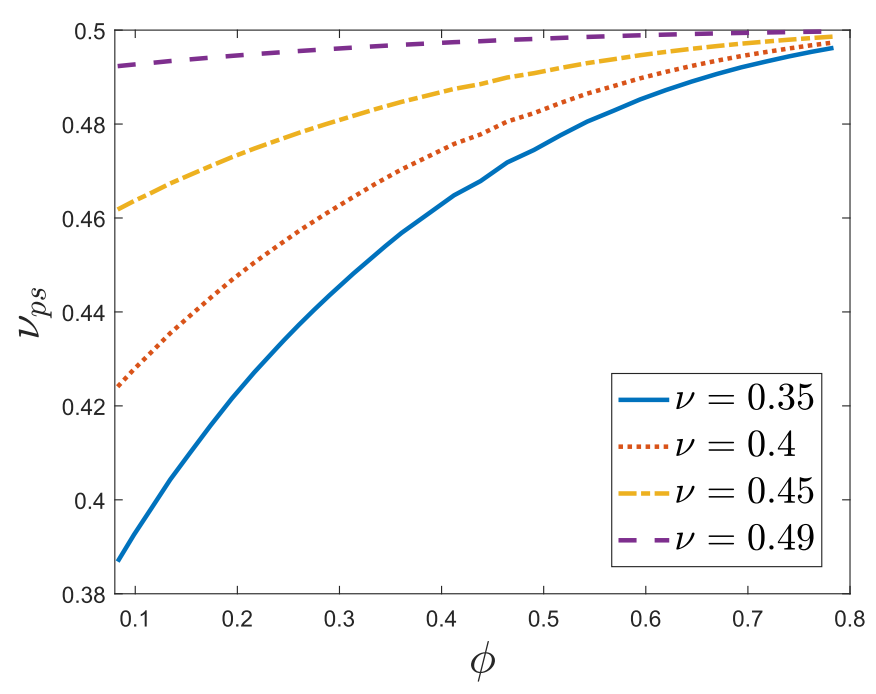

Figure 11. Undrained effective Poisson's ratio versus porosity for different Poisson's ratios of the matrix.

the model as prescribed by the asymptotic homogenization approach [21,22]. We have achieved the following crucial goals.

Firstly, we have provided a robust and accurate computational framework which can be used to obtain the three-dimensional numerical solutions of the cell problems originally deduced in [21]. We have subsequently exploited them to quantify the role of porosity, as well as solid matrix compressibility, on the macroscale elastic (Young's and shear) moduli, Poisson's ratio, hydraulic conductivity, and poroelastic parameters (Biot's modulus and Biot's coefficient). This computational framework can be readily generalized to account for highly complicated pore-scale structures, although a simple cross-shaped (cylindrical) interstitial phase is considered to limit the number of arising parameters and simultaneously account for a genuinely fully saturated, threedimensional percolation. The current analysis generalizes the pioneering computational attempt [38], where a proof of concept example of the problems reported in [21] for a fixed set of input parameters is implemented on a coarse grid.

Secondly, our results highlight the interplay between compressibility and porosity and their combined effect on the hydraulic and mechanical properties of poroelastic tissues. In particular, we have been able to quantify the effect of porosity on the macroscale compressibility of the poroelastic medium. Therefore, our predictions relate compressibility and porosity and could be exploited, in perspective, to enhance tumors type characterization (i.e. malignant vs benign [27]) on the basis of porosity (or interstitial flow) measurements. Conversely, compressibility tests could be used to formulate predictions concerning the porosity of the tissue, which is, in turn, a crucial determinant of drug transport inside the tumor mass [39].

Thirdly, we have especially focused on the analysis of the macroscale Biot's modulus. Its profile versus porosity exhibits a definite minimum for every fixed value of the matrix's Poisson's ratio, and the same Biot's modulus values can be obtained at fixed Poisson's ratio for different porosities. As Biot's modulus is related to 
the average variation of interstitial fluid volume in response to variations of the interstitial pressure, our results can be exploited to relate compressibility and fluid (and hence drug) transport in compressible tumors. Furthermore, the results can pave the way for biomimetic applications concerning the optimal design of (biological) artificial constructs with respect to the average interstitial volume. Poroelastic artificial constructs such as scaffolds and implants are actually encountered in a number of biomedical applications, such as bone ingrowth and eye implants, see, e.g. [8] and [6,7], respectively. Our framework is open to a number of improvements.

We have embraced several simplifying assumptions concerning the geometry (and periodicity of the microstructure), the constitutive relationship of the solid matrix, and also neglected the vascularization and growth of the tumor mass, as well as any possible intragrain porosity. We have assumed isotropy of the solid matrix and a cross-shaped cylindrical pore-scale structure for the sake of simplicity and to provide a robust paradigmatic platform which can be readily generalized to more complicated (tortuous) structures, as well as anisotropy of the elastic matrix, depending on the actual tissue type at hand.

The microstructure is assumed to be periodic to allow for the practical computations of the effective coefficients. This assumption can in principle be relaxed by assuming that the medium is not macroscopically uniform. This way, only local periodicity is assumed, whereas the periodic representative cell is parametrically varying with respect to the macroscale coordinate, leading to macroscopically heterogeneous coefficients. This approach [22,28] requires the solution of a periodic cell problem (of the type solved in the present manuscript) for each point of the macroscale domain, thus leading to an increase in the computational cost, although alternative strategies to reduce it are rapidly emerging in the literature, see, e.g. [40].

Generalization of the present framework to a more realistic nonlinear constitutive behavior of the solid matrix is also to be considered a cutting edge challenge in three-dimensions. In fact, appropriate threedimensional computational results in the framework of asymptotic homogenization have been only recently made available for linear problems, see [30,31,41] and this work for porous media flow, composites materials, and poroelasticity, respectively. In perspective, this work can serve as a basis towards the numerical implementation of recently emerging models in nonlinear asymptotic homogenization for poroelastic tumors [42] and composites [43].

Our work also represents the first step to build up a computational framework for multiscale appositional growth of poroelastic tumors [22]. In the latter model, the functional form of the microstructural cell problems formally coincides with that of classical poroelasticity for every fixed time point, whereas the fluid-solid interface evolves in time according to an appropriate law which is to be prescribed on the basis of the specific mechanisms driving the growth. This way, it will be possible to highlight the interplay between appositional tumor growth and elastic deformations through time evolution of the poroelastic properties of the tissue, thus better elucidating the complex interplay between tumor growth, stiffness, and porosity, see, e.g. [44].

The next natural step is the implementation of the macroscale poroelastic governing equations to capture the influence of poroelastic deformations of the solid tumor (possibly accounting for its heterogeneous microstructure informed by appropriate medical images) on the spatio-temporal profile of the fluid interstitial pressure. Predictions from such a model could provide meaningful insights into the optimization of drug injection conditions [25]. In fact, our predictions can also provide a basis in the context of poroelastic vascularized tumors [45] (by accounting for more sophisticated geometries in order to account for the blood vessels network), where the intragrain porosity (i.e. the porosity of the solid matrix itself) is explicitly taken into account, see also [46]. Solutions arising from the coupling between this model and multiscale drug transport in solid tumors, as addressed for example in $[28,47,48]$, will provide predictions that could be used to design improved anti-cancer therapies for deformable tumors.

\section{Acknowledgments}

HD and JM have been partially supported by the Ministry of Economy in Spain, under the project reference DPI2014-58885-R. HD also acknowledges financial support from the University of Glasgow.

\section{Appendix. Mesh sensitivity analysis}

The mesh setting has been chosen in order to improve the efficiency of the numerical simulations in terms of accuracy and computational cost. Since we perform a parametric analysis by varying the pore radius, the parameters that characterize the mesh setting should be geometry-dependent. This issue can be addressed by means of a mesh sensitivity analysis, thus finding appropriate relationships between the mentioned parameters and the pore radius. It is important to capture the role of the interface conditions that are actually driving the non-trivial solutions of the cell problems, as done in [31, 41, 49] in the context of elastic composites and their 


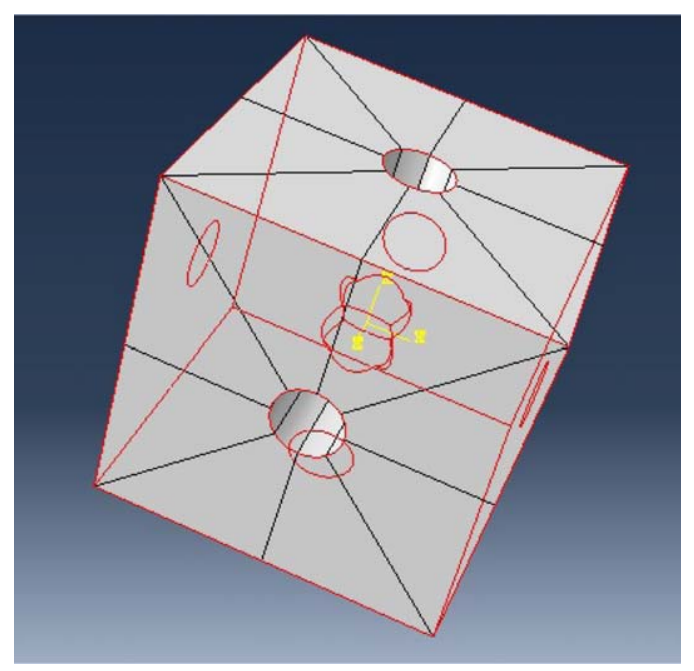

Figure A1. Two groups of edges to control the mesh size. The Circumferential and Radial edges are highlighted in red and black, respectively. By choosing the seed number for these two groups we can control the dimensions of the generated meshes.

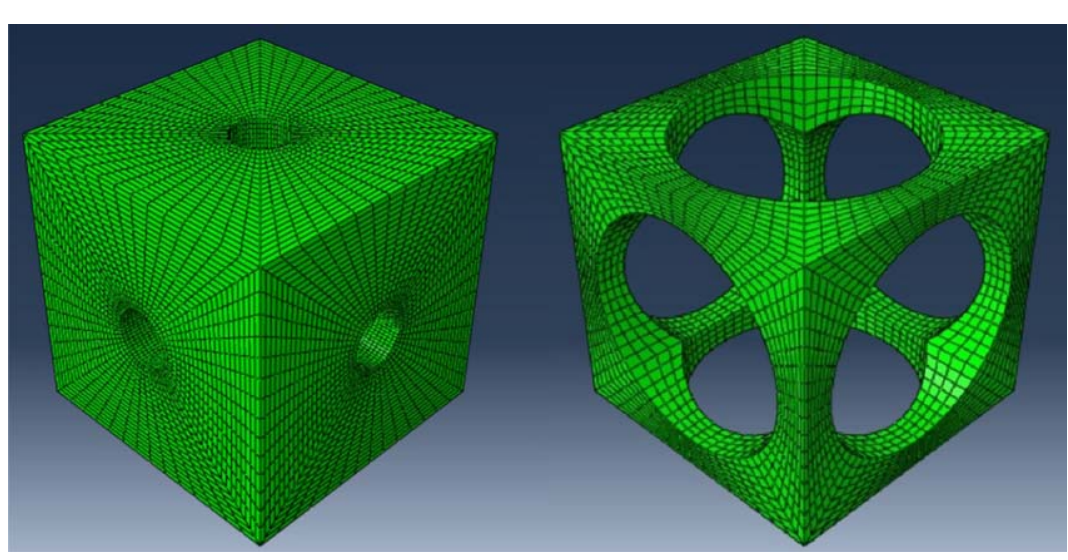

Figure A2. The generated mesh for $R=0.1$ and $R=0.4$.

application to bone and tendons modeling. In particular, the mesh around the interface should be finer than other parts far away from it. The target mesh density can be assigned by using markers that are placed along the edges of the specific region under consideration. These markers are called seed [50]. Therefore, it is convenient to divide the whole geometry to several simpler partitions. This way, by assigning the density of seeds on the edges of partitions, we can reach the desired mesh dimension in every direction. Figure A1 shows a partitioned geometry and two groups of edges that are used to control the dimension of the mesh in both radial and circumferential directions. Note that the circumferential direction of one face can be the axial direction of another face. The density of seeds on each edge (and consequently the target mesh setting) can be assigned by the parameter 'seed number' that shows the number of markers on the chosen edge. This approach is used to generate linear hexahedron, type C3D8R elements (8-node linear brick, reduced integration, hourglass control) in the solid phase.

In order to generate a geometry-dependent mesh, we relate the seed number in both directions to the radius of the pores $R$, namely

$$
\begin{gathered}
\text { Radial edges seed number }=(0.5-R) * X_{1} \\
\text { circumferential edges seed number }=R * 10 * X_{2} \text {. }
\end{gathered}
$$

We perform a parametric analysis in terms of $X_{1}$ and $X_{2}$ in order to minimize the numerical errors, as well as the computational cost. Relationships (62) and (63) ensure that the seed number is assigned consistently for both edge groups, thus obtaining a mesh density which is uniformly increasing towards the interface (see figure A2). 


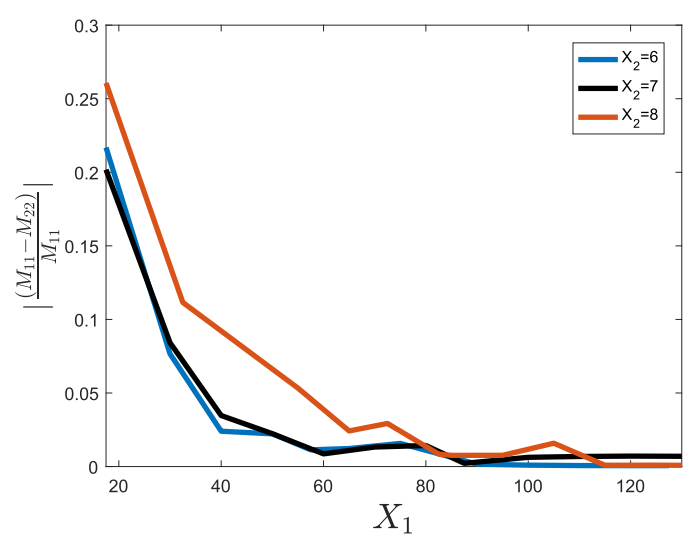

Figure A3. The results of the mesh sensitivity analysis: $\frac{M_{11}-M_{22}}{M_{11}}$ versus $X_{1}$ for the three cases $X_{2}=6,7$, and 8 in a cell with $R=0.1$.

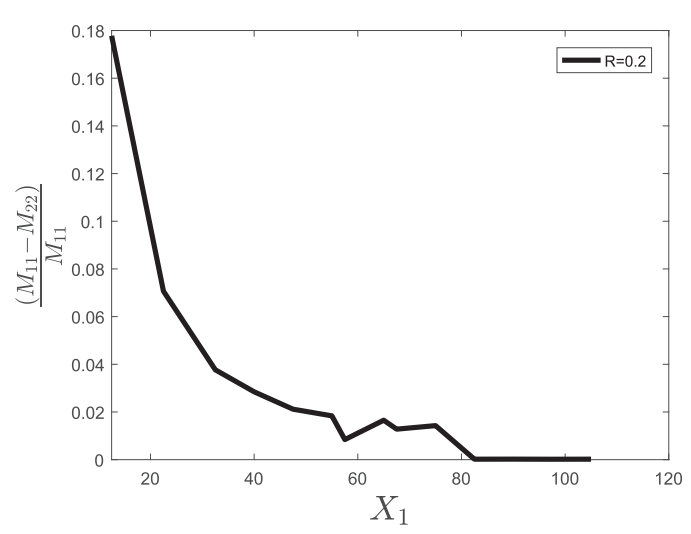

Figure A4. The results of the mesh sensitivity analysis: $\frac{M_{11}-M_{22}}{M_{11}}$ versus $X_{1}$ for the case $R=0.2$.

Since the auxiliary forth rank tensor $\mathbb{M}$ exhibits cubic symmetry then for instance

$$
M_{11}=M_{22} \text {. }
$$

The above equation is chosen to check the numerical error as the quantity $\mathrm{M}_{11}-\mathrm{M}_{22}$ should be approaching zero. Figure A3 shows the results of the sensitivity analysis for the solid phase at $R=0.1$.

In order to obtain an acceptable computational cost (total cpu time $=20 \pm 0.5 \mathrm{~s}$ ), as well accuracy of the results, the values $X_{1}=110$ and $X_{2}=7$ are chosen for the solid phase. The results of the parametric analysis conducted for the pore radius $R=0.2$ and $X_{2}=7$ are also shown in figure A4.

An alternative approach to generate mesh, based on the 'global seed size', can also be embraced. In this case, we choose an approximate size for seeds (more precisely, an approximate seed density) for the whole geometry. This approach, together with a 10-node quadratic tetrahedron element (C3D10), is used to generate the mesh for fluid phase problem. In this case, we decrease the global seed size (and therefore increase the mesh density) until the difference between two values of the hydraulic conductivity computed at increasing mesh density is less than $1 \%$.

\section{ORCID iDs}

R Penta (iD https://orcid.org/0000-0003-1202-8775

\section{References}

[1] Biot M A 1955 J. Appl. Phys. $26182-5$

[2] Biot M A 1956 J. appl. Mech. 23 91-6

[3] Biot M A 1956 The Journal of the acoustical Society of america 28 179-91 
[4] Biot M A 1962 J. Appl. Phys. 33 1482-98

[5] Wang H F 2017 Theory of Linear Poroelasticity with Applications to Geomechanics and Hydrogeology (Princeton, NJ: Princeton University Press)

[6] Chalasani R, Poole-Warren L, Conway R M and Ben-Nissan B 2007 Survey of Ophthalmology 52 145-55

[7] Jacob J T, Burgoyne C F, McKinnon S J, Tanji T M, LaFleur P K and Duzman E 1998 Journal of Biomedical Materials Research $4399-107$

[8] Karageorgiou V and Kaplan D 2005 Biomaterials 26 5474-91

[9] Cowin S C 1999 J. Biomech. 32 217-38

[10] Bottaro A and Ansaldi T 2012 Journal of Biomechanical Engineering 134084501

[11] Davit Y et al 2013 Adv. Water Resour. 62 178-206

[12] Hori M and Nemat-Nasser S 1999 Mech. Mater. 31 667-82

[13] Mavko G, Mukerji T and Dvorkin J 2009 The Rock Physics Handbook: Tools for Seismic Analysis of Porous Media (Cambridge: Cambridge University Press)

[14] Cheng A H D 2016 Poroelasticity vol 27 (Berlin: Springer)

[15] Auriault J L, Boutin C and Geindreau C 2010 Homogenization of Coupled Phenomena in Heterogenous Media vol 149 (New York: Wiley)

[16] Bakhvalov N S and Panasenko G 2012 Homogenisation: Averaging Processes in Periodic Media: Mathematical Problems in the Mechanics of Composite Materials vol 36 (The Netherlands: Springer)

[17] Holmes M H 2012 Introduction to Perturbation Methods vol 20 (New York: Springer-Verlag)

[18] Mei C C and Vernescu B 2010 Homogenization Methods for Multiscale Mechanics (Singapore: World Scientific)

[19] Bensoussan A, Lions J L and Papanicolaou G 1978 Asymptotic Analysis for Periodic Structures vol 374 (Amsterdam, New York: NorthHolland Publications)

[20] Palencia E S 1980 Non-homogeneous Media and Vibration Theory (Berlin: Springer)

[21] Burridge R and Keller J B 1981 The Journal of the Acoustical Society of America $701140-6$

[22] Penta R, Ambrosi D and Shipley R 2014 The Quarterly Journal of Mechanics and Applied Mathematics 67 69-91

[23] Laurila P and Leivo I 1993 Journal of Cell Science 104 59-68

[24] Netti P A, Berk D A, Swartz M A, Grodzinsky A J and Jain R K 2000 Cancer Research 60 2497-503

[25] Netti P A, Baxter L T, Boucher Y, Skalak R and Jain R K 1995 Cancer Research 55 5451-8

[26] Kim Y, Savellano M, Savellano D, Weissleder R and Bogdanov A 2004 Magn. Reson. Med. 52 485-94

[27] Chao T C, Lo Y F, Chen S C and Chen M F 1999 Journal of Ultrasound in Medicine 18363-70

[28] Penta R, Ambrosi D and Quarteroni A 2015 Mathematical Models and Methods in Applied Sciences 25 79-108

[29] Penta R and Gerisch A 2017 An introduction to Asymptotic homogenization Multiscale Models in Mechano and Tumor Biology (Berlin: Springer) pp 1-26

[30] Penta R and Ambrosi D 2015 J. Theor. Biol. 364 80-97

[31] Penta R and Gerisch A 2015 Computing and Visualization in Science 17 185-201

[32] Fan Z, Eichhubl P and Gale J F 2016 Journal of Geophysical Research: Solid Earth $1212798-812$

[33] Tung S and Masterlark T 2016 Journal of Geophysical Research: Solid Earth 121 3479-503

[34] Jain R K and Baxter L T 1988 Cancer Research 48 7022-32

[35] Stewart D C, Rubiano A, Dyson K and Simmons C S 2017 PloS One 12 e017756.

[36] Baker E L, Bonnecaze R T and Zaman M H 2009 Biophys. J. 97 1013-21

[37] Detournay E and Cheng A H D 1995 Fundamentals of poroelasticity Analysis and Design Methods (Amsterdam: Elsevier) pp 113-71

[38] Terada K, Ito T and Kikuchi N 1998 Comput. Methods Appl. Mech. Eng. 153 223-57

[39] Scallan J, Huxley V H and Korthuis R J 2010 Capillary fluid exchange: regulation, functions, and pathology Colloquium Lectures on Integrated Systems Physiology - From Molecules to Function vol 2 (San Rafael, CA: Morgan \& Claypool Publishers) pp 1-94

[40] Dalwadi M P, Griffiths I M and Bruna M 2015 Proc. R. Soc. A 47120150464

[41] Penta R and Gerisch A 2017 Contin. Mech. Thermodyn. 29 187-206

[42] Collis J, Brown D, Hubbard M E and O’Dea R D 2017 Proc. R. Soc. A 47320160755

[43] Ramírez-Torres A, Stefano S D, Grillo A, Rodríguez-Ramos R, Merodio J and Penta R 2018 Int. J. Non-Linear Mech. 106245-57

[44] Santagiuliana R, Pereira R C, Schrefler B A and Decuzzi P 2018 International Journal for Numerical Methods in Biomedical Engineering 34 e2935

[45] Penta R and Merodio J 2017 Meccanica 52 3321-43

[46] Arbogast T and Lehr H L 2006 Computational Geosciences 10 291-302

[47] Shipley R J and Chapman S J 2010 Bull. Math. Biol. 72 1464-91

[48] Mascheroni P and Penta R 2017 International Journal for Numerical Methods in Biomedical Engineering 33 e2857

[49] Penta R, Raum K, Grimal Q, Schrof S and Gerisch A 2016 Bioinsp. Biomim. 11035004

[50] Abaqus documentation 2016 http://130.149.89.49:2080/v2016/index.html 